\title{
A Highly Accurate Level Set Approach for Segmenting Green Microalgae Images
}

\author{
Vinicius R. Pereira Borges* ${ }^{*}$, Bernd Hamann ${ }^{\dagger}$, Thais G. Silva ${ }^{\ddagger}$, Armando A. H. Vieira ${ }^{\ddagger}$, Maria Cristina F. Oliveira* \\ Instituto de Ciências Matemáticas e de \\ Computação*, University of São Paulo \\ São Carlos, SP, Brazil \\ \{viniciusrpb,cristina\}@icmc.usp.br \\ Department of Computer Science ${ }^{\dagger}$ \\ University of California, Davis \\ Davis, CA, 95616, USA \\ hamann@cs.ucdavis.edu \\ Departamento de Botânica $\ddagger$ \\ Federal University of São Carlos \\ São Carlos, SP, Brazil \\ thais.garcia.bio@gmail.com,ahvieira@ufscar.br
}

\begin{abstract}
We present a method for segmenting 2D microscopy images of freshwater green microalgae. Our approach is based on a specialized level set method, leading to efficient and highly accurate algae segmentation. The level set formulation of our problem allows us to generate an algae's boundary curve as the result of an evolving level curve, based on computed background and algae regions in a given image. By characterizing the distributions of image intensity values in local regions, we are able to automatically classify image regions into background and algae regions. We present results obtained with our method. These results are very promising as they document that we can achieve highly accurate algae segmentations when comparing ours against manually segmented images (segmented by an expert biologist) and with results derived by other approaches covered in the literature.
\end{abstract}

Keywords-edge detection, Gaussian distribution, green microalgae, image segmentation, level set

\section{INTRODUCTION}

Freshwater green microalgae play an important role in nature and human life. These microorganisms affect water properties such as colour, odour and taste and interact with chemical compounds potentially hazardous to human or animal health. They are highly sensitive to environmental changes and therefore can signal the deterioration of ecological conditions [1], thus acting as effective indicators of water quality.

The taxonomic classification of green algae species is a highly relevant problem in biology. Typically, a taxonomist samples an algae culture for observation under a microscope and manually classifies the cells observed in the image following a pre-defined set of identification keys. The procedure is highly demanding and time-consuming, as it requires careful inspection of a diverse image collection and manual extraction of the relevant information. The overall quality of the process strongly depends on the taxonomist's skills and previous expertise, as extracting, interpreting and selecting the most suitable features to characterize the distinct families and species of algae is not straigthforward.

Previous efforts were published concerning the development of computational systems to support taxonomists in the classification of algae species. Typically, such systems embed image processing and pattern recognition algorithms that are used to capture the relevant physical properties of an image and derive an appropriate representation for further processing. Nonetheless, some systems [2] [3] employ computationally expensive manual or guided segmentation procedures that render them unfeasible for handling larger image sets. Moreover, the green algae data requires automatic segmentation techniques that are highly accurate on a wide diversity of algae shapes. Thus, we focus on segmentation techniques that can be applied to the problem of automatically extracting algae shapes from images, in order to obtain shape features that could potentially be used as input to a method performing subsequent steps required for taxonomic classification.

The main goal of image segmentation is to partition the digital image into its constitutent and disjoint regions. Algae segmentation is a particularly challenging problem, since images are acquired from a microscope and algae cells are constantly moving during the image acquisition process. Morever, the digital images obtained are characterized by low contrast due to non-uniform illumination conditions, leading to transparency of algae cells and high brightness variability accross images. The presence of artifacts is also observed, as dead cell membranes, microbes and particles, which may be mistakenly interpreted as algae cells.

From the wide diversity of image segmentation methods, techniques which incorporate variational principles have emerged as powerful tools. In a variational approach, segmentation is performed by minimizing an energy functional formulated under a homogeneity criterion of the image regions. The main references are the Mumford-Shah model [4] and the active contours [5] (also called snake model) formulations, which have been gradually modified to improve segmentation quality [6] [7] [8] or to incorporate alternative approaches for computing numerical solutions [9] [10] [11].

Deformable models, in particular, have been succesfully employed, for example, in the segmentation of organic cells [12], motion tracking [13] and fluid flow [14]. The key idea is to define a dynamic curve that evolves over the image domain towards the desired regions or object boundaries. Parametric [5] [15] approaches which represent the dynamic curve explicitly or geometric approaches which represent them implicitly [6] [16] are possible [17]. From the latter category the level set method [18] has become highly popular due to 
its natural capability of handling topological changes such as splits and breaks during curve evolution [19], unlike the parametric approaches.

The standard level set approach takes into account only the edge information of image regions as a criterion to stop curve evolution. However, it would likely produce poor segmentation results on the algae images, as the algae cells depict a wide diversity of shapes and their corresponding pixels present considerable intensity variation. Combining an appropriate regionbased term with a level set approach is likely to provide a more promising strategy in this scenario [20] [21], because the level set formulation can incorporate representative statistical models of the intensity variations observed in algae regions.

The main contribution of this paper is a technique for segmenting green algae in digital images that combines a level set formulation with Gaussian distributions first described by Rousson and Deriche [21] and Zhu et al. [20]. We represent algae regions and background by means of Gaussian distributions, whose parameters are computed from representative intensity samples of each region. We also introduce a technique based on computing image eigenvalues and eigenvectors to automatically obtain the required samples.

This paper is organized as follows: Section II presents similar approaches in the literature for segmenting algae and biological images using deformable models and establishes the motivation for this work. Section III presents the basic principles of the level set method and its relevant subsequent formulations. Section IV introduces the proposed approach based on level set and Gaussian distributions and the procedure for sampling representative image regions. Section V presents experimental results of applying the proposed strategy to a set of green algae images. Finally, Section VI summarizes conclusions and alludes to possibilities for future research.

\section{RELATED WORK}

Several strategies are reported in the literature to identify or explicitly segment cells, objects or regions of interest in biological images. Edge-based and deformable models appear as suitable strategies for handling, e.g., algae, phytoplankton and diatoms, due to their robustness to noise and efficiency to handle images formed by regions characterized by high variation in intensity.

As aforementioned, edge-based segmentation methods seek for abrupt image discontinuities that characterize region boundaries. Differential operators such as Sobel, Canny and Laplacian of Gaussian are often combined with thresholding approaches and mathematical morphology to link disconnected edges. The edge-based segmentation method by Promdaen et al. [22] applies the Sobel operator to the original algae images, with the resulting images being used as input to a Canny filter. Edge discontinuites are linked by a mathematical morphology step applied to the Canny images. Jalba et al. [23] proposed a hybrid strategy to automatically segment diatoms. Their solution combines a watershed transform based on mathematical morphology with a new marker selection scheme. The first method computes connected operators, which are then employed in the computation and selection of markers in order to prevent over-segmentation, a known drawback of watershed-based techniques.

The marine phytoplankton identification system developed by Cuiping et al. [24] segments algae cells with a regiongrowing algorithm. The system first applies a Canny edge detector to the original images, and then a morphological operator to detect algae boundaries. A thresholding with Otsu's method is applied to obtain a background patch from which mean intensity values are estimated. The regiongrowing method uses intensities from a coarse background as a stopping criterion, to automatically distribute the seeds in the image.

Whilst edge-based segmentation methods may detect edges with discontinuities, thus requiring several post-processing steps for edge-linking, deformable models are popular due to their capability of obtaining closed contours of the image objects. Moreover, such methods are usually robust under noisy conditions and can incorporate a priori knowledge about image regions.

Generally, deformable models can be associated with parametric or implicit approaches [12]. Parametric models represent the contours explicitly as parametrized curves, as in the active contour (AC) model [5] [15]. Using this representation, the parametric curve evolves from an initial position in the image domain towards the object boundaries (or the desired regions) as a result of the action of internal and external forces.

Zhang et al. [25] compared the performances of the ChanVese active contour model [26] and the 4-point approximation subdivision scheme for segmenting microalgae images. While the first technique employs a level set curve that evolves towards algae boundaries, the second relies on an iterative subdivision scheme which starts from an initial set of defined points until a polygon is obtained that approximates the algae shapes. The experiments reported show that the CV model is more accurate for detecting the algae boundaries, though initial conditions must be properly set. The iterative scheme, on the other hand, has lower computational cost and is more robust to noise. Nonetheless, it is not an edge tracking procedure, since the resulting polygon is only a rough approximation of the algae boundary.

Also employing Active Contour-based methods to identify cells in phytoplankton images, Gelzinis et al. [27] focused on improving image quality and contrast prior to segmentation. Their strategy consists of computing nine distinct images, each obtained from a specific process, such as Gabor filtering, Gaussian filtering, Sobel operator application, or other filters. Those images are binarized and their intensities added to produce a single average contour image, which is taken as reference to propagate the dynamic curves for cell segmentation. Performance of this solution is superior to that obtained when inputting the original images directly into the AC-based models.

Parametric approaches face some limitations regarding curve convergence. First, the curve needs to be positioned close to the target object, so that the forces can move the 
contour towards its boundaries. Furthermore, poor fitting of the curve and the object boundaries may occasionally happen when objects have boundaries with substantial concave pattern.

Geometric models are suitable alternatives to parametric approaches since an implicit representation of the dynamic curve can naturally handle topological changes such as splits and breaks in image objects. The level set method (LSM) [18] has been extensively employed in many image processing and computer vision problems, and its original formulation has undergone several modifications [16] [28] [26] [19] in order to improve curve stability and convergence when computing the numerical solution.

We are concerned with developing a segmentation strategy that allows us to automatically extract precise algae boundary shapes from RGB digital images obtained from a microscope. This is a first step to enable automatic feature extraction from such images, in order to devise a system to assist biologists in the taxonomic classification of such microorganisms. In handling images of a specific complex of freshwater green microalgae, identified by the biologists as the Selenestraceae family, we observed that its algae species show a wide range of shapes, e.g., from rounded, ellongated or curved single cells, as illustrated in Figure 1(a), to yet other very distinct shapes when the algae form colonies of multiple grouped cells, as depicted in Figure 1(b). This and other peculiar characteristics of the images that result from the acquisition process render the automatic segmentation task a very difficult one to handle.

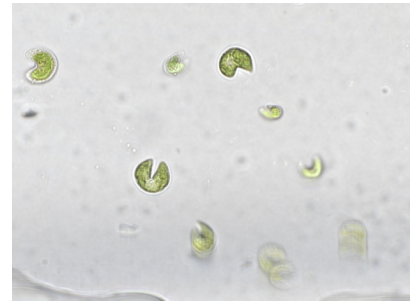

(a)

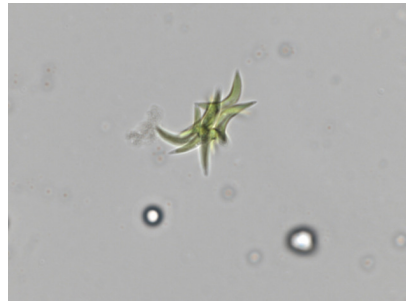

(b)
Fig. 1: Examples of green microalgae images: (a) image characterized by the presence of noise, artifacts and small objects; (b) image with the presence of colonies, showing overlap of multiple algae cells.

Because the level set formulation can incorporate both edge information and a priori region information to guide the curve evolution, we departed from the hypothesis that it is possible to devise a formulation capable of handling both the shape diversity issue and the known additional problematic characteristics of these images, such as low contrast and high intensity variation in both the algae and the background regions. As we know from the biologists that the Selenestraceae family is particularly problematic even for manual classification, we take images of its species as test cases for evaluating a proper solution to the algae segmentation problem.

We devised a novel segmentation strategy based on the level set formulation originally introduced by Rousson and Deriche [21], which employs probability distributions to statistically represent the target image regions, namely the algae cells and the background for the problem of interest here. Considering that contour shapes also convey important patterns for the algae segmentation problem [15] we incorporate an edge potential term into the energy funcional by Rousson and Deriche. Details about the standard level set method and some relevant subsequent derived formulations are described next.

\section{LEVEL SET METHOD}

Let $\Omega \subset \mathbb{R}^{m}$ be the image domain and $I: \Omega \rightarrow \mathbb{R}$ the function designed to be a digital image. Consider $\Gamma:[0,1] \times$ $[0, \infty) \rightarrow \Omega$ as the parametric curve that divides the domain in foreground $\left(\Omega_{1}\right)$ and background $\left(\Omega \backslash \Omega_{1}\right)$ regions. In the level set method, the dynamic curve is a Lipschitz function $\phi: \Omega \rightarrow$ $\mathbb{R}$, also called level set function, that can be interpreted as the zero-level of a function in higher dimension, for which:

$$
\phi(\boldsymbol{x}, t)=\left\{\begin{array}{cr}
<0 & \text { if } \boldsymbol{x} \text { is on the inside relative to } \Gamma(t) \\
0 & \text { if } \boldsymbol{x} \text { is on } \Gamma(t) \\
>0 & \text { if } \boldsymbol{x} \text { is on the outside relative to } \Gamma(t)
\end{array}\right.
$$

Here, $\phi(\boldsymbol{x}, t)$ refers to the curve position in the domain $\Omega$ at a given time step $t$. A point in the spatial domain is written here and in the following in boldface notation, i.e., the notation $\boldsymbol{x}$ represents the point $\left(x_{1}, \ldots, x_{m}\right)$. Using this compact notation simplifies many of the required equations and operators discussed in this paper. The level set function $\phi$ evolves through the domain $\Omega$ according to a speed function $F$, given by the level set equation:

$$
\frac{\partial \phi}{\partial t}+F|\nabla \phi|=0
$$

knowing that $\phi(\boldsymbol{x}, 0)=\phi_{0}(\boldsymbol{x})$ is the initial position of the curve in $\Omega$. The level set function is usually defined by means of a signed distance function, such as the Euclidean distance:

$$
\phi(\boldsymbol{x}, t)=\left\{\begin{array}{rr}
-d_{E}(\boldsymbol{x}, \Gamma(t)) & \begin{array}{r}
\text { if } \boldsymbol{x} \text { is on the inside } \\
\text { relative to } \Gamma(t)
\end{array} \\
d_{E}(\boldsymbol{x}, \Gamma(t)) & \begin{array}{r}
\text { if } \boldsymbol{x} \text { is on the outside } \\
\text { relative to } \Gamma(t)
\end{array}
\end{array}\right.
$$

As for $F$, the usual choices are the mean curvature [16], in which the speed is defined by the curvature values of curve points, and the geometric term [15] in which an edge potential function is used to stop curve evolution at objects boundaries.

Eq. (1) must be solved numerically and the level set function may gradually degrade along successive time steps, due to numerical instabilities. This problem is handled using the reinitializing level set equation, described by:

$$
\frac{\partial \phi}{\partial t}=\operatorname{sign}\left(\phi_{0}\right)(1-|\nabla \phi|)
$$

in which $\operatorname{sign}\left(\phi_{0}\right)$ is computed as:

$$
\operatorname{sign}(\phi(\boldsymbol{x}, t))=\left\{\begin{array}{cl}
-1 & \text { if } \phi(\boldsymbol{x}, t)<0 \\
0 & \text { if } \phi(\boldsymbol{x}, t)=0 \\
+1 & \text { if } \phi(\boldsymbol{x}, t)>0
\end{array}\right.
$$


Although the level set method is suitable for segmenting algae, its standard formulation is highly sensitive to the initial positioning of the curve. Some authors have incorporated region-based terms into the level set formulation, or have employed sophisticated optimization schemes [9] [10], in order to address this problem.

The method introduced by Chan and Vese [26] assumes that an image consists of statistically homogeneous regions, and settles the constant case of the Mumford-Shah functional using the level set formulation, which aims to minimize the following energy functional:

$$
\begin{array}{r}
F_{C V}\left(c_{1}, c_{2}, \phi\right)=\lambda_{1} \int_{\Omega}\left(I(\boldsymbol{x})-c_{1}\right)^{2} H(\phi(\boldsymbol{x})) d \boldsymbol{x}+ \\
\lambda_{2} \int_{\Omega}\left(I(\boldsymbol{x})-c_{2}\right)^{2}(1-H(\phi(\boldsymbol{x}))) d \boldsymbol{x}+ \\
\nu \int_{\Omega} \delta(\phi(\boldsymbol{x}))|\nabla \phi(\boldsymbol{x})| d \boldsymbol{x}+\rho \int_{\Omega} H(\phi(\boldsymbol{x})) d \boldsymbol{x} .
\end{array}
$$

in which $\rho, \lambda_{1}, \lambda_{2}$ and $\nu$ are positive parameters acting as weights for their respective terms. Constants $c_{1}$ and $c_{2}$ are statistical representations (mean intensities) of the foreground and background regions. $\delta(z)=\frac{d}{d z} H(z)$ is the Dirac function. Heaviside functions $H(\phi)$ allow us to represent geometrical quantities and properties of the image domain, using

$$
H(z)= \begin{cases}1 & \text { if } z \geq 0 \\ 0 & \text { if } z<0\end{cases}
$$

However, some of the underlying assumptions do not apply to green algae images, which present considerable intensity variation even within the algae cells. We propose capturing such algae patterns by computing Gaussian distributions that characterize the image regions and incorporating such information into the level set method. This strategy, described in detail in the following section, has been inspired by Rousson and Deriche's method [21].

\section{Proposed Method}

Rousson and Deriche (RD) [21] formulated an energy functional based on the level set method which uses probability distributions to describe image regions statistically [20] [28]. For that purpose, it is assumed that the intensities at each point $\boldsymbol{x} \in \Omega$ are independent and identically distributed by the same random process and the image regions are statistically independent.

Let $P_{1}\left(I(\boldsymbol{x}) \mid \theta_{1}\right)$ and $P_{2}\left(I(\boldsymbol{x}) \mid \theta_{2}\right)$ be the probability distributions of the foreground and background regions, respectively. Taking the same level set formulation adopted by ChanVese, but employing the probability distributions to model image regions, Rousson and Deriche proposed minimizing the following energy functional:

$$
\begin{array}{r}
\min _{\phi,\left\{\theta_{1}, \theta_{2}\right\}}\left\{F_{R D}\left(\phi,\left\{\theta_{1}, \theta_{2}\right\}\right)=\int_{\Omega}|\nabla H(\phi)|\right. \\
-\lambda \int_{\Omega} H(\phi(\boldsymbol{x})) \log \left(P_{1}\left(I(\boldsymbol{x}) \mid \theta_{1}\right)\right) d \boldsymbol{x} \\
\left.-\lambda \int_{\Omega}(1-H(\phi(\boldsymbol{x}))) \log \left(P_{2}\left(I(\boldsymbol{x}) \mid \theta_{2}\right)\right) d \boldsymbol{x}\right\} .
\end{array}
$$

in which the region parameters $\left\{\theta_{1}, \theta_{2}\right\}$ are estimated according to an optimization scheme further described elsewhere [21]. Although RD's method performs well on the green algae images, its formulation does not incorporate edge information, which provides important patterns for characterizing distinct algae shapes.

Motivated by existing strategies [15] [28], we incorporate an edge potential function into the first term of RD's energy functional, in order to reduce the diffusion process on region boundaries. The underlying rationale is to preserve region edges as much as possible in order to favor accurate identification of algae shapes. Finally, the energy functional used in our proposed method is

$$
\begin{array}{r}
\min _{\phi}\left\{F_{P M}\left(\phi,\left\{\theta_{1}, \theta_{2}\right\}\right)=\int_{\Omega} g|\nabla H(\phi)|\right. \\
-\lambda \int_{\Omega} H(\phi(\boldsymbol{x})) \log \left(P_{1}\left(I(\boldsymbol{x}) \mid \theta_{1}\right)\right) d \boldsymbol{x} \\
\left.-\lambda \int_{\Omega}(1-H(\phi(\boldsymbol{x}))) \log \left(P_{2}\left(I(\boldsymbol{x}) \mid \theta_{2}\right)\right) d \boldsymbol{x}\right\} .
\end{array}
$$

The first term of the functional in Eq. (6) is the length of the contour $\Gamma$ and the two remaining terms refer to the cost of assigning each domain point inside and outside the contour. The minimization problem defined by Eq. (6) is solved by deriving and solving the Euler-Lagrange equations using a gradient descent scheme in relation to $\phi$ :

$$
\frac{\partial \phi}{\partial t}=\operatorname{div}\left(g \frac{\nabla \phi}{|\nabla \phi|}\right)+\lambda \log \left(P_{2} / P_{1}\right)
$$

in which $g$ is a positive boundary potential, usually chosen to be a decreasing function of the image gradient. Generally, $g$ is computed as:

$$
g(|\nabla I|)=\frac{1}{1+\beta|\nabla I|^{2}}
$$

in which $\beta$ is a positive constant that controls the gradient influence of the first term in Eq. (6). The dynamic curve evolves according to the log-likelihood test defined by the second term in Eq. (7).

We chose multivariate Gaussians to describe the image regions. The distribution parameters $\theta_{1}=\left\{\mu_{1}, \Sigma_{1}\right\}$ and $\theta_{2}=\left\{\mu_{2}, \Sigma_{2}\right\}$ are the mean and the covariance matrix of the algae and the background regions, respectively. The associated Gaussian distributions are computed as: 


$$
\begin{aligned}
& P_{i}\left(I(\boldsymbol{x}) \mid\left\{\mu_{i}, \Sigma_{i}\right\}\right)= \\
& \frac{1}{\sqrt{(2 \pi)^{m}\left|\Sigma_{i}\right|}} \exp \left(-\frac{1}{2}\left(I(\boldsymbol{x})-\mu_{i}\right)^{T} \Sigma_{i}^{-1}\left(I(\boldsymbol{x})-\mu_{i}\right)\right)
\end{aligned}
$$

in which the values of the parameters $\theta_{1}$ and $\theta_{2}$ are estimated once before the curve evolution, and kept fixed during the optimization process of Eq. (6). Parameter estimation consists of sampling pixel intensities of both image regions, as described next.

\section{A. Automatic Sampling}

The extended method requires sampling intensities of the foreground and the background regions in order to estimate the parameters of the probability distributions modeling the target regions. The quality of the probability distributions estimated depends on an effective sampling procedure. We introduce an automatic sampling strategy that relies on computing a binary mask from the eigenvalues of the covariance matrix of the original RGB image.

The sampling technique has a local aspect since it computes the covariance matrix of the color channels in relation to each domain point. Thus, it is possible to represent the local image structure and identify subtle details in the image patterns [29].

First, we compute the local mean value relative to each image domain point:

$$
\begin{gathered}
\mu_{L}(\boldsymbol{x})=\frac{1}{|\Omega|} \int_{\Omega} I(\boldsymbol{x}-\boldsymbol{y}) d \boldsymbol{y} . \\
A(\boldsymbol{x})=I(\boldsymbol{x})-\mu_{L}(\boldsymbol{x}) .
\end{gathered}
$$

The following step requires us to compute a local covariance matrix, given by:

$$
C(\boldsymbol{x})=A(\boldsymbol{x})^{T} A(\boldsymbol{x}) .
$$

Finally, we compute the eigenvalues and eigenvectors of the covariance matrix $C(\boldsymbol{x})$ relative to each domain point:

$$
V^{-1} C(\boldsymbol{x}) V=D
$$

in which $V$ is the matrix of eigenvectors and $D$ is a diagonal matrix of the eigenvalues of $C(\boldsymbol{x})$, given by $v=$ $\left\{D_{1,1}, \ldots, D_{m, m}\right\}$. Considering that eigenvalues are computed for each pixel, they can be represented as $m$ images, each one depicting the algae image properties from multiple perspectives. An inspection of the eigenvalue images substantiated our choice to pick the third eigenvalue image (green channel) as representative, as it better captures the image characteristics.

The mask which defines the pixels as associated with either algae or background regions is obtained by thresholding the selected eigenvalue image using its mean intensity value. In the resulting binary image the algae-related pixels are white whereas the background pixels have low intensities. For performance reasons we decided to sample only $10 \%$ of the background pixels.
Figure 2 illustrates the sampling steps, from the initial stage of obtaining the eigenvalue images to the probability distribution parameter estimation stage. Figure 2(a) shows the original green algae image. Figures 2(b), 2(c) and 2(d) depict the images constructed from the first, second and third eigenvalues of each image domain point. Figure 2(e) shows the binary image obtained after thresholding using the chosen eigenvalue image. Figure 2(f) illustrates the obtained patches for sampling intensities from the target regions of interest, the algae (shown in red) and the background (in green).

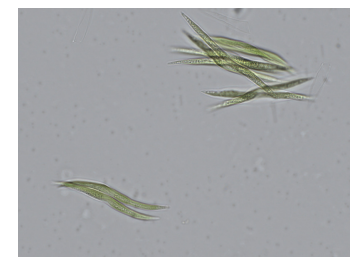

(a)

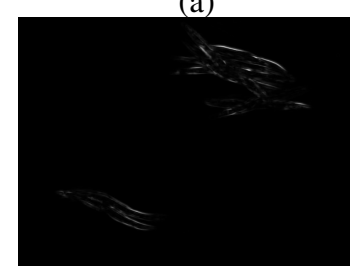

(c)

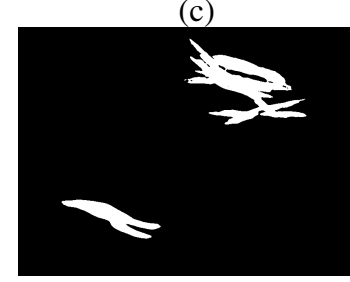

(e)

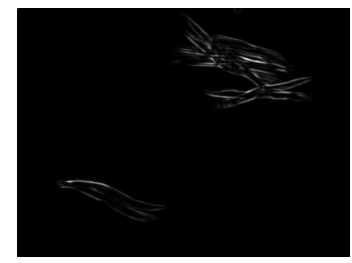

(b)

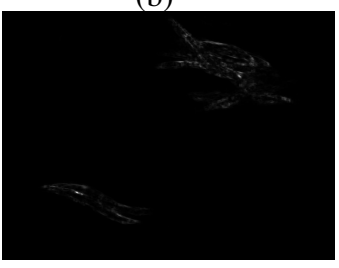

(d)

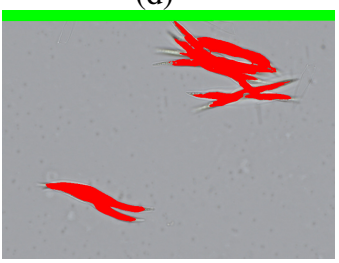

(f)
Fig. 2: Examples illustrating the image sampling procedure: (a) original RGB image; (b-c-d) eigenvalue images obtained for each RGB channel; (e) generated binary mask; (f) mask used for sampling, in which the red patch is related to algae pixels and the green patch with the background.

In summary, our proposed algorithm consists of these major steps:

1) Compute the eigenvalue image as previously described and sample pixel intensities from both the algae and the background regions.

2) Estimate the Gaussian probability distributions parameters $\theta_{1}=\left\{\mu_{1}, \Sigma_{1}\right\}$ and $\theta_{2}=\left\{\mu_{2}, \Sigma_{2}\right\}$, given the algae and background region samples, respectively.

3) Compute the multivariate Gaussian distributions $P_{1}$ and $P_{2}$ for those regions, according to Eq. (9).

4) Initialize $\phi_{0} \approx \phi(\boldsymbol{x}, 0)$ using Eq. (2).

5) Use a finite difference approach [30] to compute the numerical solution of Eq. (7) in relation to the level set function $\phi$. We assume that $\Omega \subset \mathbb{R}^{2}$ and $\boldsymbol{x}=(x, y)$, so the level set function and the digital images are discretized as bidimensional $[M, N]$ matrices, in which 
$\phi(\boldsymbol{x}) \approx \phi_{i, j}$ for $i=1, \ldots, M$ and $j=1, \ldots, N$. The level set curve evolution stops once it reachs the algae boundaries.

6) Once $\phi$ converges, we generate the binary image by thresholding the final level set as $\phi(\boldsymbol{x})<0$.

7) In the binary image we keep the regions whose perimeters exceed 50 (measured in units defined by the uniform spacing of the underlying implicitly defined image grid) and perform a dilation morphological operation using a disk structuring element with radius of size 2 to smooth the algae boundaries.

In Section $\mathrm{V}$ we present results obtained when applying our proposed method on a particular set of green algae images.

\section{EXPERIMENTAL RESULTS}

We have evaluated the performance and the effectiveness of the proposed method for segmenting green algae. A set composed by 44 images of green algae depicting different species of the Selenestraceae families complex was used for the tests. These images have a resolution of $600 \times 800$ and are quantized in 8 bits per color channel. The parameters in Equations (7) and (8) were set to $\lambda=0.2$ and $\beta=0.0005$ by means of visual observation of the results. We performed some experiments using several combinations of parameters values and chose those which produced the best accuracy rates.

The goal of the first experiment was to verify the method's dependence on initial conditions, i.e., how the initial level set curve positioning affects the final segmentation result. The sequences in Figures 3(c-e-g-i) and 3(d-f-h-j) show two test cases, each one considering a distinct initial position of the level set curve. Figures 3(c-d) show the initial position of the level set $\phi$. Figures 3(e-f) show the intermediate states of $\phi$, and Figures 3(g-h) illustrate the final positions of $\phi$ after 1,620 iterations. Figures 3(i-j) present the binary image representing the algae segmentation. As the proposed method does not optimize the parameter distributions during the minimizing process of the energy function of Eq. (6), it is less sensitive to initial conditions when compared with RD's model.

In the second part of the experiments, we have evaluated our method by comparing the accuracy of the obtained segmentations with those manually segmented by biologists, refered to as ground-truth (GT) images. To that end, we have used the binary image obtained by thresholding the final contour $\phi$, in which algae regions and cells are associated with the white pixels and the background pixels have intensity zero. The accuracy (Acc) is defined as:

$$
A c c=\frac{T P+T N}{T P+T N+F P+F N},
$$

in which $T P$ (true positive) refers to the pixels labeled as belonging to algae regions in both segmentation and GT. FP (false positive) are the pixels labeled as belonging to algae regions in the segmentation, but as non-algae pixels in GT. $T N$ (true negative) are the pixels labeled as non-algae in both segmentation and in the GT. $F N$ (false negative) refers to the pixels labeled as non-algae in the segmentation, but are

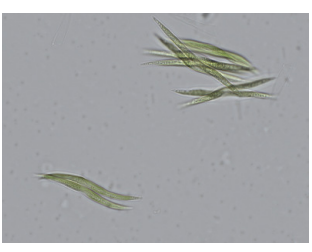

(a)

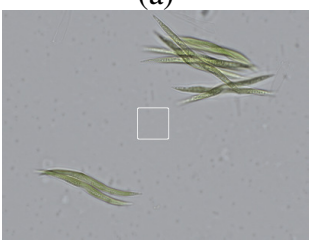

(c)

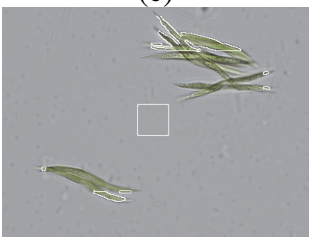

(e)

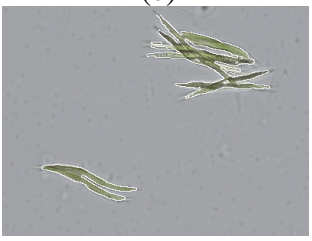

(g)

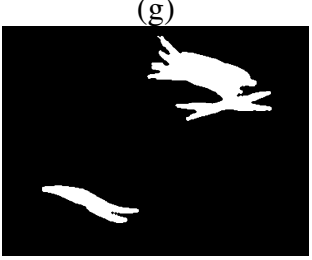

(i)

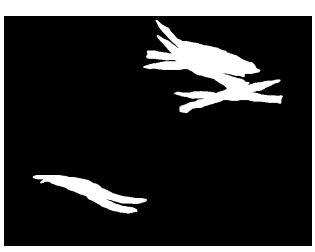

(b)

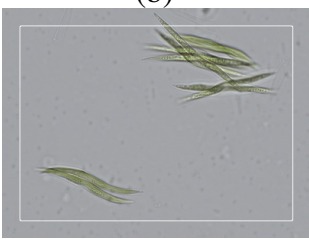

(d)

(f)

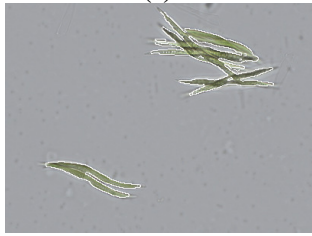

(h)

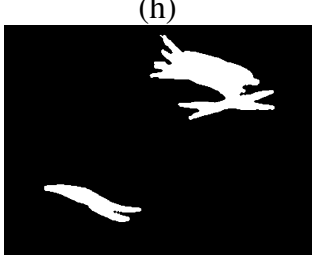

(j)

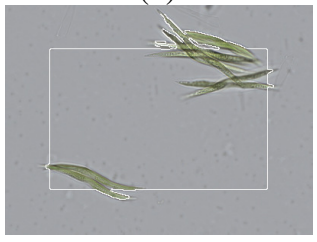

Fig. 3: Level set evolution from two distinct initial positions: (a) original RGB image; (b) segmentation after thresholding final $\phi$; (c-d) initial $\phi(\boldsymbol{x}, 0)$; (e-f) intermediary state of $\phi ;(\mathrm{g}-\mathrm{h})$ after convergence of $\phi$.

actually pixels belonging to algae regions in the GT image. We measure the average accuracy for an image set by averaging the accuracy values computed for each image.

For the given image set, Table I presents the average accuracy rates and the standard deviation (std) obtained by the proposed method, by the conventional level set method and by Rousson and Deriche's approach. In the conventional level set method, we employed the speed term proposed by [15] in Eq. (1) and after each time iteration, the level set curve was reinitialized according to Eq. (2). In turn, we derived Rousson and Deriche's method by minimizing the energy functional in Eq. (5) in relation to $\phi$ and the distribution parameters $\left\{\theta_{1}, \theta_{2}\right\}$. In both methods, we initialized the level set curve in the same position and discretized the associated energy functionals by the Finite Difference method. Parameters of 
each method have been adjusted after visual observation of the results and considering the higher accuracy rates in the obtained segmentations. The proposed method achieved higher accuracy rates, emphasizing that the modification introduced into RD's formulation is effective for segmenting green algae images. Analysing the standard deviation (std) rates, the proposed method is also more consistent, obtaining satisfactory segmentations in the majority of test cases. Moreover, results show that the segmented algae shapes are highly similar to those obtained manually by biologists.

TABLE I: Average accuracy rates and standard deviation (std)

\begin{tabular}{|l|c|c|}
\hline Segmentation techniques & accuracy & std \\
\hline 1. Proposed Method & $\mathbf{0 . 9 6 2}$ & $\mathbf{0 . 0 0 9}$ \\
2. Conventional level set [18] [15] & 0.938 & 0.0116 \\
3. Rousson and Deriche's [21] & 0.951 & 0.0209 \\
\hline
\end{tabular}

Figure 4 illustrates a test case comparing the segmentation resulting from the proposed model with the ground-truth. Figure 4(a) shows the original RGB image, while Figures 4(b), 4(c) and 4(d) depict the curve evolution in its initial, intermediate and final positions (after 1,520 time iterations). Figure 4(e) presents the binary image of the resulting segmentation and Figure 4(f) shows the GT image. The algae cells were succesfully identified, as their overall shapes in the colony were preserved. It is worth noting that the level set function was placed away from the algae colony and correctly evolved to the desired boundaries, confirming that the proposed model is relatively insensitive to changes of initial conditions.

Figure 5 presents a difficult segmentation case where the algae cells have distinctly varying intensities. The original image is illustrated in Figure 5(a) and the subsequent Figures 5(b), 5(c) and 5(d) show the level set evolution from an initial to the final position, after 1,510 iterations. Figures 5(e) and 5(f) respectively, show the obtained segmentation and the GT image. The level set surrounded correctly the algae cells due to the appropriate representation of image regions as Gaussian distributions, which captures the intensity patterns according to the sampling procedure. This test case achieved accuracy equal to 0.959 .

In general, it was observed that the multivariate Gaussians are effective statistical representations of the image regions. Such models can capture the patterns and details of each region, and the sampling procedure ensures that distinguishing distributions are computed between them, yielding to a faster evolution of the dynamic curve towards the desired image regions. Unlike Rousson and Deriche's model, the distribution parameters in our model are computed a priori and remain fixed along the level set function optimization, leading to faster convergence and less sensitivity to initial conditions.

The proposed method also has limitations. One is that it detects small particles which have intensity patterns similar to those of the green algae. This problem is handled by selecting only the larger regions obtained after convergence of the dynamic curve and the binarization procedure, so that regions associated with unwanted particles are discarded.

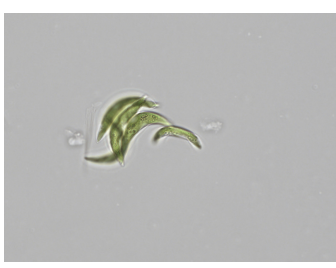

(a)

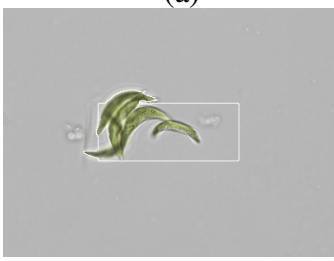

(c)

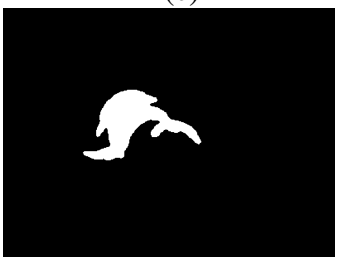

(e)

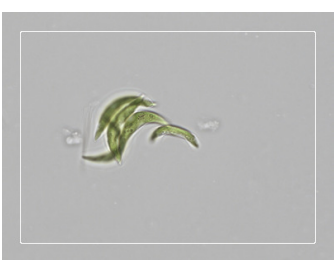

(b)

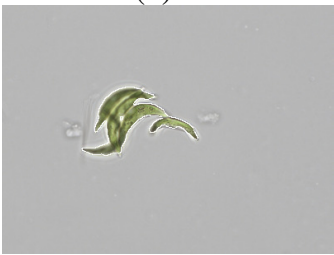

(d)

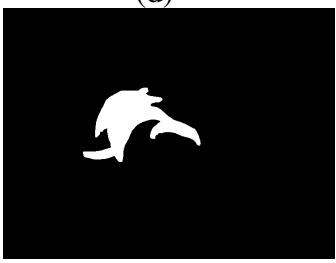

(f)
Fig. 4: Segmentation of algae colony with accuracy of 0.965 : (a) original RGB image; (b) initial $\phi(\boldsymbol{x}, 0)$; (c) intermediary state of $\phi$; (d) final level set function after 1,520 iterations; (e) segmentation after thresholding $\phi$; (f) ground-truth image.

Also, the method's energy functional is non-convex due to the optimization space of Heaviside functions, meaning that infinite solutions are valid representations of a given optimal $\Omega_{1}$. The practical implication is that the method is weakly sensitive to initial conditions and local optimal solutions are obtained by computing numerical solutions to the EulerLagrange equations associated with gradient descent schemes.

\section{CONCLUSIONS}

We have introduced a technique for segmenting green algae images based on a level set approach combined with Bayesian principles. The image regions, i.e., the algae cells and the background, are described by multivariate Gaussian distributions computed prior to the curve evolution process. The key idea is to capture the intensity variation that may arise in algae cells for an accurate segmentation.

The proposed method incorporates edge and region information, since it is based on Rousson and Deriche's functional and incorporates an edge potential function to preserve boundaries during the segmentation. Therefore, the dynamic curve evolves from an initial position towards the algae cells, considering $a$ priori region properties, such as intensity variation and texture, and edge information for shape preservation.

Experimental results have shown that the proposed method achieves high segmentation accuracy when compared with ground-truth segmentations provided by the biologists. Moreover, it also produced better segmentation accuracy rates than 


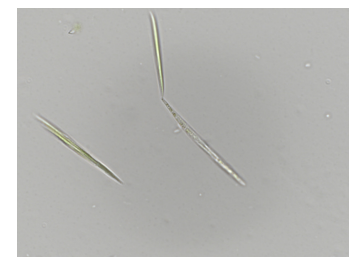

(a)

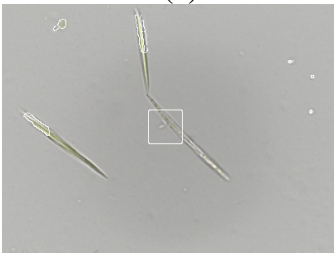

(c)

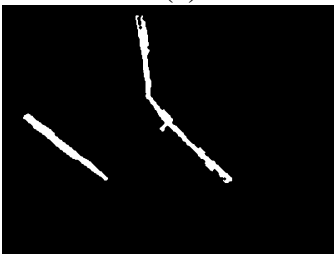

(e)

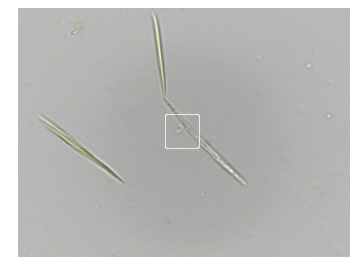

(b)

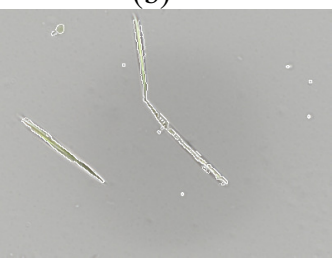

(d)

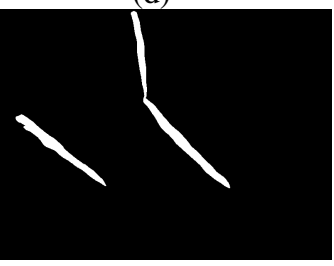

(f)
Fig. 5: Segmentation case with accuracy of 0.959: (a) original RGB image; (b) initial $\phi(\boldsymbol{x}, 0)$; (c) intermediary state of $\phi$; (d) final level set function after 1,510 iterations; (e) segmentation after thresholding final $\phi$; (f) ground-truth image.

the conventional level set method and the original approach by Rousson and Deriche.

Future work would be concerned with applying our method to other biological image processing problems with similar characteristics. Exploring alternative energy minimization approaches might also be a good direction for further research.

\section{ACKNOWLEDGMENT}

The authors acknowledge the support of the State of Sao Paulo Research Funding Agency (FAPESP), procs. 2011/22749-8, 2012/00269-7, 2013/26647-0 and the Brazilian Federal Funding Agency (CNPq), proc. 305696/2013-0.

\section{REFERENCES}

[1] P. McCormick and J. Cairns, "Algae as indicators of environmental change," Journal of Applied Phycology, vol. 6, no. 5, pp. 509-526, 1994.

[2] K. Embleton, C. Gibson, and S. Heaney, "Automated counting of phytoplankton by pattern recognition: a comparison with a manual counting method," Journal of Plankton Research, vol. 25, no. 6, pp. 669-681, 2003.

[3] S. Brasch, L. Linsen, and G. Fuellen, "Vanlo-interactive visual exploration of aligned biological networks," BMC Bioinformatics, vol. 10, no. 1 , p. 327,2009

[4] D. Mumford and J. Shah, "Optimal approximations by piecewise smooth functions and associated variational problems," Communications on Pure and Applied Mathematics, vol. 2, pp. 577-685, 1989.

[5] M. Kass, A. Witkin, and D. Terzopoulos, "Snakes: Active contour models," International Journal of Computer Vision, vol. 1, no. 4, pp. 321-331, 1988.

[6] S. Osher and R. P. Fedkiw, "Level set methods: an overview and some recent results," Journal of Computational Physics, vol. 169, no. 2, pp. 463-502, 2001.
[7] T. Andersson, G. Lathen, R. Lenz, and M. Borga, "Modified gradient search for level set based image segmentation," IEEE Transactions on Image Processing, vol. 22, no. 2, pp. 621-630, 2013.

[8] X. Yang, X. Gao, D. Tao, X. Li, and J. Li, "An efficient mrf embedded level set method for image segmentation," IEEE Transactions on Image Processing, vol. 24, no. 1, pp. 9-21, 2015.

[9] X. Bresson, S. Esedoglu, P. Vandergheynst, J.-P. Thiran, and S. Osher, "Fast global minimization of the active contour/snake model," Journal of Mathematical Imaging and Vision, vol. 28, no. 2, pp. 151-167, 2007.

[10] K. Zhang, L. Zhang, H. Song, and W. Zhou, "Active contours with selective local or global segmentation: a new formulation and level set method," Image and Vision computing, vol. 28, no. 4, pp. 668-676, 2010.

[11] N. P. van Dijk, K. Maute, M. Langelaar, and F. Van Keulen, "Level-set methods for structural topology optimization: a review," Structural and Multidisciplinary Optimization, vol. 48, no. 3, pp. 437-472, 2013.

[12] J. P. Bergeest and K. Rohr, "Efficient globally optimal segmentation of cells in fluorescence microscopy images using level sets and convex energy functionals," Medical image analysis, vol. 16, no. 7, pp. 14361444, 2012.

[13] Y. Yu, S. Zhang, J. Huang, D. Metaxas, and L. Axel, "Sparse deformable models with application to cardiac motion analysis," Information Processing in Medical Imaging, pp. 208-219, 2013.

[14] E. O. Kung, A. S. Les, C. A. Figueroa, F. Medina, K. Arcaute, R. B. Wicker, M. V. McConnell, and C. A. Taylor, "In vitro validation of finite element analysis of blood flow in deformable models," Annals of Biomedical Engineering, vol. 39, no. 7, pp. 1947-1960, 2011.

[15] V. Caselles, R. Kimmel, and G. Sapiro, "Geodesic active contours," International Journal of Computer Vision, vol. 22, pp. 61-79, 1997.

[16] R. Malladi, J. A. Sethian, and B. C. Vemuri, "Shape modeling with front propagation: A level set approach," IEEE Transactions on Pattern Analysis and Machine Intelligence, vol. 17, no. 2, pp. 158-175, 1995.

[17] C. Xu, A. Yezzi Jr, and J. L. Prince, "On the relationship between parametric and geometric active contours," 33th Asilomar Conference on Signals, Systems and Computers, vol. 1, pp. 483-489, 2000.

[18] S. Osher and J. A. Sethian, "Fronts propagating with curvaturedependent speed: algorithms based on hamilton-jacobi formulations," Journal of Computational Physics, vol. 79, no. 1, pp. 12-49, 1988.

[19] C. Li, C. Xu, C. Gui, and M. D. Fox, "Distance regularized level set evolution and its application to image segmentation," IEEE Transactions on Image Processing, vol. 19, no. 12, pp. 3243-3254, 2010.

[20] S. C. Zhu and A. L. Yuille, "Region competition: unifying snakes, region growing, energy/bayes/mdl for multi-band image segmentation," IEEE Transactions on Pattern Analysis and Machine Intelligence, vol. 18, pp. 884-900, 1996.

[21] M. Rousson and R. Deriche, "A variational framework for active and adaptative segmentation of vector valued images," Workshop on Motion and Video Computing, pp. 56-61, 2002.

[22] S. Promdaen, P. Wattuya, and N. Sanevas, "Automated microalgae image classification," Proc Computer Science, vol. 29, pp. 1981-1992, 2014.

[23] A. C. Jalba, M. H. F. Wilkinson, and B. T. M. Roerdink, "Automatic segmentation of diatom images for classification," Microscopy Research and Technique, vol. 65, no. 1, pp. 72-85, 2004

[24] S. Cuiping, Y. Chenhui, L. Huizhen, and K. Lin, "A system for identification of marine phytoplankton," 2nd International Conference on Signal Processing Systems, vol. 3, pp. V3-426, 2010.

[25] L. Zhang, Z. Luo, B. Wang, and J. Zhang, "Comparative study of cv active contour model and subdivision for micro algae image segmentation," International Conference on Electric Information and Control Engineering, pp. 1241-1244, 2011.

[26] T. F. Chan and L. A. Vese, "Active contours without edges," IEEE Transactions on Image Processing, vol. 10, no. 2, pp. 266-277, 2001.

[27] A. Gelzinis, E. Vaiciukynas, M. Bacauskiene, A. Verikas, S. Sulcius, R. Paskauskas, and I. Olenina, "Boosting performance of the edge-based active contour model applied to phytoplankton images," IEEE 13th International Symposium on Computational Intelligence and Informatics, pp. 273-277, 2012.

[28] N. Paragios and R. Deriche, "Geodesic active regions and level set methods for supervised texture segmentation," International Journal of Computer Vision, vol. 46, no. 3, pp. 223-247, 2002.

[29] X. Zhu and P. Milanfar, "Automatic parameter selection for denoising algorithms using a no-reference measure of image content," IEEE Transactions on Image Processing, vol. 19, no. 12, pp. 3116-3132, 2010.

[30] W. F. Ames, Numerical methods for partial differential equations. Academic Press, 2014. 